\title{
Narrow-band X-ray polarizing filters
}

\author{
A. Martindale ${ }^{a}$, N.P. Bannister ${ }^{a}$, K.D.M. Harris ${ }^{b}$, G.A. Solan ${ }^{c}$, S.P. Collins ${ }^{d}$, Y. Champouret $^{c}$, \\ V.K. Muppidi ${ }^{b}$, G.W. Fraser ${ }^{a}$ and M.Roy ${ }^{e}$ \\ ${ }^{a}$ Space Research Centre, Department of Physics and Astronomy, University of Leicester, \\ University Road, Leicester, LE1 7RH, UK; \\ ${ }^{b}$ School of Chemistry, Main Building, Cardiff University, Park Place, Cardiff, Wales, UK; \\ ${ }^{c}$ Department of Chemistry, George Porter Building, University of Leicester, University Road, \\ Leicester, LE1 7RH, UK; \\ ${ }^{d}$ Diamond Light Source Ltd, Rutherford Appleton Laboratory, Chilton, Didcot, Oxon OX11 \\ OQX, UK \\ ${ }^{e}$ Department of Physics and Astronomy, University of Leicester, University Road, Leicester, \\ LE1 7RH, UK
}

\begin{abstract}
We review past and current attempts to measure X-ray polarization in celestial sources and describe research activity into a new family of materials which have been shown to exhibit linear dichroism at X-ray wavelengths. Such materials could add a polarimetry capability to the high energy resolution detectors proposed for future, high effective area, X-ray astrophysical observatories such as Constellation-X and XEUS. They have the potential to achieve useful minimum detectable polarization values for a number of sources in a sensible exposure time with XEUS.
\end{abstract}

Keywords: X-ray polarization, Astronomy, Polarimetry, XAFS

\section{INTRODUCTION}

In recent years, almost all areas of X-ray instrumentation have seen rapid development, allowing astronomers to gain an improved understanding of celestial X-ray sources and the processes involved in driving them. These advancements are embodied by the successes of Chandra ${ }^{1}$ and XMM-Newton, ${ }^{2}$ where new mirror and detector technologies enabled unprecedented spatial and energy resolution, providing a significant expansion in our understanding of astrophysical X-ray sources. Major new advances can also be expected in astronomical X-ray spectroscopy, through the deployment of cryogenically cooled detectors. Elsewhere, the study of transient objects and time variability is being addressed by new X-ray all sky monitor proposals such as the UK-led Lobster mission, ${ }^{3}$ while the Swift observatory (launched November 2004) leads the quest to understand explosive Gamma Ray Bursts.

In contrast, astronomical X-ray polarimetry remains as unexplored now as it was 30 years ago. Conventional X-ray polarimetry relies on the Bragg reflection ${ }^{4}$ or Thomson scattering ${ }^{5}$ of photons. Although polarimeters based on these physical principles have flown on sounding rockets, ${ }^{6}$ and on the Ariel- $5^{7}$ and OSO- $8^{8}$ satellites, to date only one unambiguous measurement of polarisation in a cosmic X-ray source has been made - that of $19 \%$ linear polarisation at $2.6 \mathrm{keV}$ for the Crab Nebula. ${ }^{9}$ The low photon fluxes received from astronomical objects, combined with narrow bandwidth (for Bragg crystal polarimeters ${ }^{10,11}$ ) or low scattering efficiency (for Thomson scatterers) have rendered polarisation observations insensitive to all but the brightest sources.

In this paper we describe the difficulties of practical X-ray polarimetry (XRP) and review past and current instrumentation to exploit the polarization of celestial sources. We explain the concept of narrow bandwidth

Further author information: (Send correspondence to A.M., K.D.M.H. or G.A.S)

A.M.: E-mail: adrian.martindale@star.le.ac.uk, Telephone: +44 (0)116 2522650

K.D.M.H.: E-mail: HarrisKDM@Cardiff.ac.uk, Telephone: +44 (0)29 20870133

G.A.S.: E-mail: gas8@le.ac.uk, Telephone: +44 (0)116 252 2096: 
dichroic filters, and describe how such devices could add a polarimetry capability to the cryogenic focal planes of the next generation of X-ray observatory. This work was undertaken as part of a one year feasibility study funded by the Research councils of the UK (RCUK) basic technology programme. The goal of this study was to investigate whether these filters are capable of delivering a simple, compact, low mass X-ray polarimeter for future X-ray astronomical observatories.

\section{POLARISATION OF X-RAYS}

The degeneracy in predictions from competing astrophysical models, when constrained only by photometric and spectroscopic observations, can be broken by measuring the degree $(P)$ and angle $(\theta)$ of polarization. Despite similar predictions for the current observables, the models often predict differences in $P$ and $\theta$, and also differences in how their values change when the observer wishes to discriminate the polarisation as a function of time and/or photon energy. X-ray polarimetry therefore promises the realization of the next milestone in X-ray astronomy by addressing many areas of interest, but where the most exciting and influential results from a new detector technology are usually those which are unexpected. In this section we review some current problems in astrophysics which would benefit from an efficient polarimeter.

If emitted in a thermal process, photons will be unpolarized (assuming they do not undergo any polarization dependent process along the light path - e.g. scattering or transiting a region of intense magnetic field). Conversely, if photons are produced in a non-thermal process, they will have some intrinsic polarization whose degree and direction can be used to identify the driving emission process. The measurement of $19 \%$ linear X-ray polarization in the Crab nebula ${ }^{9}$ was used to confirm the synchrotron nature of the observed X-ray spectrum which was long suspected owing to its power law nature. However, it was not until the polarisation was measured that it was possible to exclude a complex thermal spectrum. This demonstrates the power of polarimetry to discriminate between astrophysical models.

Many emission processes can cause polarization of photons. Weisskopf et. al. review a number of such mechanisms, concentrating on the use of polarimetry to understand neutron star systems; ${ }^{12}$ identifying the variation in polarization as a function of pulsar phase as a prime example of where X-ray polarimetric data could distinguish between competing astrophysical models. The authors also describe a method of obtaining possible observational evidence for vacuum birefringence - an exotic effect of quantum electrodynamics (QED). This theory predicts that in the presence of a strong magnetic field, the refractive index of a vacuum is different for orthogonally polarized photons. Consequently, certain models of pulsar emission predict variation in the polarization dependence as a function of pulsar phase and photon energy. Weisskopf et. al. predict a $\sim 10^{\circ}$ phase lag between optical and X-ray polarization angle as a function of pulsar period for the Crab pulsar owing to this effect. A number of other authors have reviewed the possible impact of X-ray polarimetry on different astrophysical processes demonstrating the broad interest in polarimetry across the astrophysics community. ${ }^{10,13-15}$

\section{X-RAY POLARIMETERS}

Soffitta et. al. (2003) review past and present instrumentation for X-ray astrophysical polarimetry. ${ }^{11}$ The authors show that Bragg crystal polarimeters are insensitive to all but the brightest sources because of the narrow band, dispersive technique that they employ. Also, the Thompson scattering technique, in which measuring the azimuthal distribution of photons scattered from a low atomic number medium yields $P$ and $\theta$, is shown to be inefficient. The origin of this inefficiency is twofold; first, the intrinsically weak scattering in the medium means that only a small fraction of incident photons are subject to the modulation, the dependence of scattering efficiency on atomic number (z) means that low-z materials must be used. Second is the acceptance of photons at angles other than normal incidence in order to increase the number of photons incident on the detector. The authors state that a perfectly collimated device would have perfect modulation. However, to improve photon statistics, it is necessary to accept photons arriving within a discrete range of angles, this reduces modulation factor.

The intrinsically weak nature of past polarimeters means that a better method of measuring beam polarisation is needed, recent polarimeters have relied on the polarization dependence of photoelectron emission to convert beam polarization into a measurable quantity. When an X-ray is incident onto a material, an electron is emitted 
if the photon energy is greater than the work function of the material. This is the photoelectric effect, and the preferred direction of emission of the photoelectron is in the plane of the electric field of the photon.

Electrons can also be excited into higher bound states in a molecule. Although this is not generally the case for isolated atoms or ions; in a molecule, electrons can be excited from the bonding orbital into higher, bound states such as the anti-bonding orbital. In this case the electronic structure around the molecule provides the "allowed states" into which the electron can be excited. Therefore, the absorption probability of the photon can be a function of the polarization of the photon and the local electronic structure. It should be noted that photo-ionization is still possible and is a special case of this more general description, where the free electron is excited into the infinite band of allowed states outside of the atomic potential. Both photo-ionization and excitation to higher, bound states can potentially provide the active element of a practical X-ray polarimeter.

This effect has been investigated in two proposed practical polarimeters where either the efficiency of the photocathode of an $\mathrm{MCP}^{16}$ or the presence of split pixel events in a $\mathrm{CCD}^{17}$ image yield the mean polarization direction of a beam. These effects proved too weak and inefficient for a practical instrument owing to the falling efficiency of the CCD at the high energies needed to excite split pixel events, or the difficulty of discriminating between a true polarization modulation of the beam and spurious systematic effects in the MCP system.

\subsection{Novel X-ray Polarimeters}

In 2001, Costa et. al. proposed a micro-structured proportional counter which could directly image the emission direction of photoelectrons in the absorbing gas volume. ${ }^{18}$ In this design, a histogram of emission directions is produced. The maximum intensity in the histogram corresponds to the mean polarization direction of the incident beam and the depth of the sinusoidal modulation curve gives the polarized fraction. Jahoda et. al. describe an evolution of this device, using a time projection chamber rather than a gas electron multiplier to image the photoelectrons. ${ }^{19}$ These divices are proposed for inclusion on the next generation of great observatories, XEUS and Constellation- $X(C o n-X)$.

Bannister et. al. (2006) describe a novel way to exploit the polarization dependence of photo-excitation of core level electrons which yields a polarization sensitive transmission filter. ${ }^{20}$ The polarization sensitivity arises from the difference in transmission of a family of materials which exhibit dichroism in narrow $(\sim 10 \mathrm{eV})$ energy bands close to atomic absorption edges, where the electron is excited into a bound, molecular orbital. The simplicity of such a device makes it an ideal instrument for future X-ray observatories as it decouples the polarization sensitivity from the intrinsic efficiency of the detector. When combined with the high efficiency and energy resolution of the cryogenic instrumentation under development for future telescopes such as XEUS and $C o n-X$, these filters may offer a simple and compact method of measuring X-ray polarisation in a large number of astrophysical sources, finally ending the 35 year hiatus in this field.

\section{DICHROIC FILTERS}

In 1967, Auton ${ }^{21}$ showed that a wire grid of period $d$ will effectively polarize light of wavelength $\lambda$ if $d \leq \lambda / 2$. At visible wavelengths, polarization can be detected using highly ordered materials such as Polaroid H-sheet, a commercially available polymer sheet which is used in LCD displays and sunglasses. H-sheet consists of long chain polyvinyl alcohol molecules which are stretched during manufacture to ensure that all polymer chains are uniformly aligned. Doping these chains with iodine makes them conduct along their length, imposing a preferred axis on the material in which light polarized parallel to the chains is efficiently absorbed, while light polarized in a direction perpendicular to the chains is transmitted.

Despite the wavelength dependence of the wire grid dichroic polarizers, which should preclude the operation of materials such as H-sheet at X-ray wavelengths, Collins (1997) reports the successful analysis of polarization of $33.17 \mathrm{keV} \mathrm{X}$-rays using ordinary $\mathrm{H}$-sheet. ${ }^{22}$ The underlying physics explaining this result is very different from that which governs optical wavelength polarization.

The nanoscopic structure of $\mathrm{H}$-sheet was identified as the cause for the difference in transmission of the filter as a function of the azimuthal angle between the beam polarization and the principal axis of the polarizer. The long chains of doped polyvinyl alcohol (PVA) molecules produce a different electronic structure in two perpendicular axes; the number of allowed molecular electronic states must therefore differ in these two axes. 

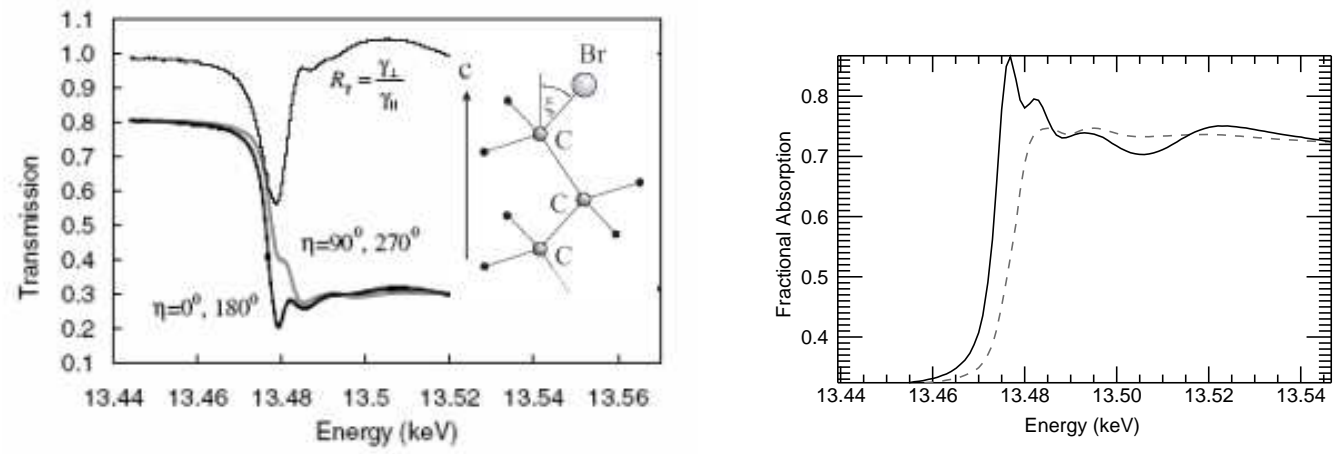

Figure 1. Transmission spectra from Collins $2002^{23}$ for a dibromoalkane - urea compound the upper curve is the parameter $R_{\gamma}$ which is the ratio of the perpendicular and parallel attenuation coefficients for the polariser. The black curve is for the case where the polarisation is parallel to the polariser axis and the gray curve is for polarisation perpendicular to this axis(left). Our results for a bromoadamantane/thiourea compound, taken at the Daresbury Synchrotron Radiation Source (SRS) beamline 16.3 are shown where the solid line is for polarisation parallel to the polarizer axis and the dashed line is for polarisation perpendicular to this axis (right).

As the most probable direction of ejection of a photoelectron is parallel to the polarization vector of the photon, the absorption probability differs in these two principal axes, leading to a difference in the X-ray absorption coefficient. The energy bands into which the electron can be exited are discrete (and narrow) and therefore the change in transmission occurs over a narrow range of energies, leading to fine structure in the absorption spectra. This is known as X-ray absorption fine structure (XAFS) and is a function of polarization state, meaning that within energy bands of $\sim 10 \mathrm{eV}$ the filer is dichroic (i.e. it shows different transmission according to whether the source is polarized parallel or perpendicular to the principal axis of the filter).

A material designed with highly ordered and different electronic structure in two perpendicular axes is therefore expected to produce an efficient polarizer in $\sim 10 \mathrm{eV}$ bands around the absorption edge of the atom under excitation. To design such a material, single crystals with highly ordered bonding structure are required, which give rise to a preferred transmission axis in the material. Our initial attempts at producing such materials, based on the previous experience of $\mathrm{KDMH}$ and SC, concentrated on inclusion compounds, where the molecules are constrained to have a particular orientation owing to the fact that they are held within a tunnel-like host substance. The host matrix is used to confine molecules of interest into a structure where their chemical bonds remain coaligned with the axes of the host tunnel. The hosts of interest here are Urea and Thiourea, which crystalise into tunnel structures, these can be engineered to contain diverse guest molecules of interest. The host material acts to reduce the transmission of the filter without adding to the dichroic response and although this is not a problem for high energy photons (as the absorption in the host is small) at lower energies, absorption in the host dominates.

The initial attempts to make polarising filters concentrated on a dibromoalkane/urea compound ${ }^{23}$ (Figure 1 [left]), this was shown to be an unoptimised material because the bonds in the guest molecule did not align perfectly with the tunnel axis. Bromoadamantane/thiourea exhibits perfectly aligned bonding with the tunnel axis and Figure 1 [right] shows that this is a much better polarisation analyser as the strength of the differential effect is increased by the better bond alignment. A completely new approach, where coordination chemistry is used to constrain directionality in the crystal is possible but will be described elsewhere.

\section{ASTROPHYSICAL INSTRUMENT}

The efficiency of polarimeters is defined in terms of two figures of merit: the modulation factor $(M)$ and minimum detectable polarisation $(M D P)$. The modulation factor is defined as;

$$
M=\frac{\left(C_{\perp}-C_{\|}\right)}{\left(C_{\perp}+C_{\|}\right)}
$$


Here $C_{\perp}$ and $C_{\|}$are the count rates experienced by the detector with the polarimeter axis perpendicular and parallel to the beam polarization vector for a $100 \%$ polarised beam. The minimum detectable polarisation $M D P$ is defined by Novick et. al. ${ }^{13}$ as the $3 \sigma$ or $99 \%$ confidence limit of a polarimeter;

$$
M D P=P(3 \sigma)=\frac{3}{M S}\left(2\left(\frac{S+B}{T}\right)\right)^{1 / 2} .
$$

In this expression, $P(3 \sigma)$ is the $99 \%$ confidence limit of the measurement, defining the $M D P$ for a source, $S$ is the signal counting rate, $B$ is the background count rate, $T$ is the observing time and $M$ is the modulation factor as defined above. If a polarisation $P>P(3 \sigma)$ is measured, it is $99 \%$ certain that the effect is real and not a statistical anomaly.

For astrophysicaly useful measurements, a polarimeter must be capable of measuring $1 \%$ polarisation for a number of celestial sources. This limit is imposed by the fact that a typical polarisation at other wavelengths (e.g. optical and radio) is of order $1 \%$, and by the need to resolve polarisation in a large number of different targets.

We have shown ${ }^{20}$ that the minimum detectable polarization for a number of sources could compare favorably to the micro-structured proportional counter instrument ${ }^{24}$ based on the XEUS effective area and assuming the detectors are capable of resolving the edges of interest. We showed $M D P$ values of a few percent for four active galactic nuclei (AGN) at $2.8 \mathrm{keV}$ assuming the success of the bromine polarisers could be replicaeted at $\mathrm{Cl}-\mathrm{K}$ $(2.8 \mathrm{keV})$, we also showed much better than $1 \% M D P$ for these sources if a filter could be made to operate at lower energies $(\sim 1 \mathrm{keV}$ and below).

Optimisation of the thickness of the filter, specifically to minimise $M D P$, could significantly improve the filter performance even for the high energy edges such as Br-K. However, we expect to get $\leq 3 \% \mathrm{MDP}$ at 13.5 $\mathrm{keV}$ for the Crab nebula with existing (unoptimised) filters based on the Br-K edge in a $10^{5} \mathrm{~s}$ observarion with XEUS. Although theoretically by optimising the filter such that there is zero transmission in one axis and the modulation factor is $100 \%$, in practise a tradeoff is needed between modulation factor and photon counting statistics, this will be investigated in future work. It should also be noted that although $3 \% M D P$ seems rather modest, the peak intensity of the spectrum lies significantly below the $13.5 \mathrm{keV}$ edge, and also the effective area of the telescope is relatively low at this high energy meaning that the number of incident photons is low and photon statistics will be poor. The goal of the next phase of study is to push the dichroic filters to lower energy, where the telescope effective area and the intensity from the source is significantly higher. Future work will include a more comprehensive study of a number of different types of sources and the sensitivity of a dichroic filter to their polarisation. We are confident that in extending the dichroic response to lower energies, the MDP will be competitive.

\section{CONCLUSIONS}

Based on the results of our one year feasibility study, we conclude that it is possible to engineer dichroic materials to perform as transmissive polarising filters at X-ray wavelengths. Despite the narrow energy bands, the development of cryogenic spectrometers, capable of resolving the $\sim 10 \mathrm{eV}$ dichroic bandwidth of the filter offers an efficient method of making such measurements. Although predictions are only made for XEUS, the results are scalable to $C o n-X$ as the $M D P$ is governed by photon statistics and therefore scales in proportion to the observing time and the effective area. Work is ongoing and shows significant promise in extending the energies of interest to lower, more useful energy absorption edges.

\section{ACKNOWLEDGEMENTS}

This work was funded through the RCUK Basic Technology programme under grant EP/D00490X/1. The authors would like to acknowledge the support of Mina Golshan and David Laundy of the Daresbury SRS for all their help. AM would like to acknowledge the support of the STFC for funding his studentship. 


\section{REFERENCES}

1. M. C. Weisskopf, H. D. Tananbaum, L. P. Van Speybroeck, and S. L. O'Dell, "Chandra X-ray Observatory (CXO): overview," in Proc. SPIE Vol. 4012, p. 2-16, X-Ray Optics, Instruments, and Missions III, Joachim E. Truemper; Bernd Aschenbach; Eds., J. E. Truemper and B. Aschenbach, eds., Presented at the Society of Photo-Optical Instrumentation Engineers (SPIE) Conference 4012, pp. 2-16, July 2000.

2. D. H. Lumb, H. Eggel, R. Laine, and A. J. Peacock, "X-ray Multimirror Mission: an overview," in Proc. SPIE Vol. 2808, p. 326-337, EUV, X-Ray, and Gamma-Ray Instrumentation for Astronomy VII, Oswald H. Siegmund; Mark A. Gummin; Eds., O. H. Siegmund and M. A. Gummin, eds., Presented at the Society of Photo-Optical Instrumentation Engineers (SPIE) Conference 2808, pp. 326-337, Oct. 1996.

3. G. W. Fraser, A. N. Brunton, N. P. Bannister, J. F. Pearson, M. Ward, T. J. Stevenson, D. J. Watson, B. Warwick, S. Whitehead, P. O'Brian, N. White, K. Jahoda, K. Black, S. D. Hunter, P. Deines-Jones, W. C. Priedhorsky, S. P. Brumby, K. N. Borozdin, T. Vestrand, A. C. Fabian, K. A. Nugent, A. G. Peele, T. H. Irving, S. , S. Eckersley, I. Renouf, M. Smith, A. N. Parmar, I. M. McHardy, P. Uttley, and A. Lawrence, "LOBSTER-ISS: an imaging x-ray all-sky monitor for the International Space Station," in Proc. SPIE Vol. 4497, p. 115-126, X-Ray and Gamma-Ray Instrumentation for Astronomy XII, Kathryn A. Flanagan; Oswald H. Siegmund; Eds., K. A. Flanagan and O. H. Siegmund, eds., pp. 115-126, Jan. 2002.

4. R. A. Gowen, B. A. Cooke, R. E. Griffiths, and M. J. Ricketts, "An upper limit to the linear X-ray polarization of SCO X-1," Monthly notices of the Royal Astronomical Society 179, pp. 303-310, May 1977.

5. J. R. Lemen, G. A. Chanan, J. P. Hughes, M. R. Laser, R. Novick, I. T. Rochwarger, M. Sackson, and L. J. Tramiel, "A Solar Flare X-Ray Polarimeter for the Space Shuttle," Solar Physics 80, pp. 333-+, Oct. 1982.

6. R. Novick, M. C. Weisskopf, R. Berthelsdorf, R. Linke, and R. S. Wolff, "Detection of X-Ray Polarization of the Crab Nebula," ApJ 174, pp. L1+, May 1972.

7. R. E. Griffiths, B. A. Cooke, A. Peacock, K. A. Pounds, and M. J. Ricketts, "The Leicester X-ray crystal spectrometer on Ariel V and some early results on CAS A, TYCHO and SCO X-I," MNRAS 175, pp. 449460, June 1976.

8. R. Novick, M. C. Weisskopf, E. H. Silver, H. L. Kestenbaum, K. S. Long, and R. S. Wolff, "The OSO-8 Mosaic Graphite Stellar X-Ray Polarimeter," in Advances in Space Exploration, K. A. van der Hucht and G. Vaiana, eds., pp. 127-+, 1978.

9. M. C. Weisskopf, E. H. Silver, H. L. Kestenbaum, K. S. Long, and R. Novick, "A precision measurement of the X-ray polarization of the Crab Nebula without pulsar contamination," ApJ 220, pp. L117-L121, Mar. 1978.

10. P. Meszaros, R. Novick, A. Szentgyorgyi, G. A. Chanan, and M. C. Weisskopf, "Astrophysical implications and observational prospects of X-ray polarimetry," ApJ 324, pp. 1056-1067, Jan. 1988.

11. P. Soffitta, L. Baldini, R. Bellazzini, A. Brez, E. Costa, G. di Persio, L. Latronico, N. Omodei, L. Pacciani, and G. Spandre, "Techniques and detectors for polarimetry in X-ray astronomy," Nuclear Instruments and Methods in Physics Research A 510, pp. 170-175, Sept. 2003.

12. M. C. Weisskopf, R. F. Elsner, D. Hanna, V. M. Kaspi, S. L. O'Dell, G. G. Pavlov, and B. D. Ramsey, "The prospects for X-ray polarimetry and its potential use for understanding neutron stars," ArXiv Astrophysics e-prints, Nov. 2006.

13. R. Novick, "Stellar and Solar X-Ray Polarimetry," in IAU Colloq. 23: Planets, Stars, and Nebulae: Studied with Photopolarimetry, T. Gehrels, ed., pp. 262-+, 1974.

14. R. Blandford, E. Agol, A. Broderick, J. Heyl, L. Koopmans, and H.-W. Lee, "Compact objects and accretion disks," in Astrophysical Spectropolarimetry, CUP, J. Trujillo-Bueno, F. Moreno-Insertis, and F. Sánchez, eds., pp. 177-223, 2002.

15. T. Kallman, "Astrophysical motivation for X-ray polarimetry," Advances in Space Research 34, pp. 2673$2677,2004$.

16. G. W. Fraser, J. E. Lees, and J. F. Pearson, "Measurement of vectorial effects in the X-ray and UV photoemission from CsI: A novel polarimeter for soft X-ray astronomy," Nuclear Instruments and Methods in Physics Research A 284, pp. 483-508, Dec. 1989.

17. A. D. Holland, A. D. T. Short, G. W. Fraser, and M. J. L. Turner, "The X-ray polarisation sensitivity of CCDs," Nuclear Instruments and Methods in Physics Research A 355, pp. 526-531, Feb. 1995. 
18. E. Costa, P. Soffitta, R. Bellazzini, A. Brez, N. Lumb, and G. Spandre, "An efficient photoelectric X-ray polarimeter for the study of black holes and neutron stars," Nature 411, pp. 662-665, June 2001.

19. K. Jahoda, K. Black, P. Deines-Jones, J. E. Hill, T. Kallman, T. Strohmayer, and J. H. Swank, "An X-ray Polarimeter for Constellation-X," ArXiv Astrophysics e-prints, Jan. 2007.

20. N. P. Bannister, K. D. M. Harris, S. P. Collins, A. Martindale, P. S. Monks, G. Solan, and G. W. Fraser, "Dichroic Filters For Astronomical X-Ray Polarimetry," Experimental Astronomy, pp. 27-+, Sept. 2006.

21. J. P. Auton, "Infrared transmission polarizers by photolithography," Applied optics 6, pp. 1023-+, June 1967.

22. S. P. Collins, "Polaroid H-sheet as a polarizer for $33 \mathrm{keV}$ X-rays," Nuclear Instruments and Methods in Physics Research Section B: Beam Interactions with Materials and Atoms 129, pp. 289-296, July 1997.

23. S. P. Collins, D. Laundy, K. D. M. Harris, B. M. Kariuki, C. L. Bauer, S. D. Brown, and P. Thompson, "Xray linear dichroism in an \&alpha;,\&omega; -dibromoalkane/urea inclusion compound and its application to polarization analysis of magnetic diffraction," Journal of Physics: Condensed Matter 14(1), pp. 123-134, 2002.

24. E. Costa, P. Soffitta, G. di Persio, M. Feroci, L. Pacciani, A. Rubini, R. Bellazzini, A. Brez, L. Baldini, L. Latronico, N. Omodei, and G. Spandre, "X-ray Astronomical Polarimetry in the XEUS Era," in XEUS studying the evolution of the hot universe, G. Hasinger, T. Boller, and A. N. Parmer, eds., pp. 235-+, 2003. 